\title{
THE MEASURING POSITION DESIGNED TO DETERMINE THE METROLOGICAL PROPERTIES OF AIR GAUGES
}

\author{
Michal Jakubowicz'1, Janusz Derezynski ${ }^{1}$ \\ 1 Faculty of Mechanical Engineering and Management, Institute of Mechanical Engineering, Poznan University of \\ Technology, 5 M. Sklodowska-Curie Square,60-965 Poznan,Poland, e-mail:michal.jakubowicz@put.poznan.pl
}

Received: 2017.09.14

Accepted: 2017.11.01

Published: 2017.12.05

\begin{abstract}
This article describes the measurement system designed in order to determine the metrological properties of air gauges. The said scientific study makes it possible to determine the static $p_{k}=f(s)$ and flow $q_{v}=f(s)$ characteristics. It consists of three modules: a mechanical module, a control and register data module and a special software module. Apart from the possibility of determining the static and flow characteristics, the presented study makes it possible to measure the temperature in the duct that supplies the compressed air to the transducer as well as in the measuring chamber. The above-mentioned measurement system makes it possible to determine the pressure applied on the surface measured by an air stream coming from the nozzle. Apart from a detailed description of a test station and the software, the article also contains sample results of tests performed on air gauges.
\end{abstract}

Keywords: air gauges, static characteristics, metrology

\section{INTRODUCTION}

The metrology of geometrical figures is very important as regards the manufacturing processes. Because of the fact that the products must comply with high standards, it has become necessary to continuously streamline and improve the measurement techniques. New and more precise processing techniques require new appliances and measuring devices, which would guarantee more precision as regards the measurements as well as decrease the measurement time, the cost of measurement devices and their service. At the same time, many manufacturers tend not only to control the chosen details, but all the elements of an assembly line, which eliminates the risk of manufacturing a faulty product.

Out of many methods of measuring geometrical figures, pneumatic methods are a very significant group. They make use of air gauges [2, $3,7,8,9,11]$. The history of pneumatic length measuring gauges dates back to the 1920s [12].
Since then, the interest in these methods has been changing depending on the development of electronics or microprocessor technologies. Since the late 1980s, another surge in the interest in pneumatic length measuring gauges may be observed. It is connected with the universal accessibility of inexpensive and precise pressure transducers, which make it possible to convert a pneumatic signal to an electronic one and, then to assess the measurement results with the help of a proper software [10, 11].

The usability of pneumatic transducers and the accessibility of technical means of production bring about an interest in researching the metrological properties of air gauges. In order to carry out these studies, a measuring position to determine the metrological parameters of air gauges was designed and built. This measuring position makes it possible to determine the static characteristic $p_{k}=f(s)$ and the flow characteristic $q_{v}=f(s)$. The station takes measurements in an automatic cycle and enables the researcher to 
take measurements of many types of transducers, which use various configurations of measuring and inlet nozzles as well as measuring chambers. The station designed for similar purposes was discussed in articles $[1,4,5,6,8]$. When designing the structure of the new system, the attention was drawn particularly to the possibility of automatic registration of metrological parameters of air gauges. The system was also upgraded - it was possible to measure the temperature in the measuring chamber and at the intake of air. The possibility of determining the measurement pressure applied by an air stream coming out of a measuring nozzle to the surface being measured was also introduced.

The results yielded using the discussed measuring position enable the researcher the obtain information about the metrological properties of an air gauge in question.

\section{THE THEORETICAL BACKGROUND}

The flapper-nozzle systems are used in pneumatic measurements of geometrical values. In this system the compressed air comes out of the gap $s$ that was created between the nozzle and the measured object. The change in the distance between the two elements causes a change in parameters of the air that comes out. The measurement of the change of parameters of the compressed air is used to measure this distance $[1,3,5]$. Depending on which parameter of the compressed air that flows through the transducer will be used for measurement, there are pressure transducers, flow transducers and current transducers [3].

The air gauge (Fig. 1) is composed of an inlet nozzle 1 , a measuring chamber 2 and measuring nozzle 3. A greater number of measuring nozzles (a pneumatic bore gauge) often exists in application solutions $[1,3,5]$.

The outflow of air from a measuring nozzle 3 is restricted by a flapper 4 (a measured surface), which is distant from the head by distance s. In industrial measuring systems the flapper area constitutes the measured object. In air gauges the inlet and measuring nozzles are most often of circular cross-section and their diameters are $d_{w}$ and $d_{k}$ respectively. The measuring pressure (cascade pressure) $p_{k}$, whose value changes depending on the function of the size the measured gap $s$, exists in the measuring chamber. The course of this function is dependent on the geometry of the nozzles $[3,5]$.

The basic metrological characteristic of air gauges is the static characteristic $p_{k}=f(s)$ describing the relation between the measurement pressure $p_{k}$, measured in a measuring chamber shown in the function of the distance between the head of the measuring nozzle and the flapper (the measured surface) - the measuring gap $s[3,5]$.

The sensitivity characteristic turns out to be useful for assessing the linearity. The sensitivity characteristic shows the change in linearity in the function of the width of the gap (clearance) $s$ of the static characteristic. Fig.2. shows the sample static characteristic $p_{k}=f(s)$ and the sensitivity characteristic $|K|=g(s)$.

a)

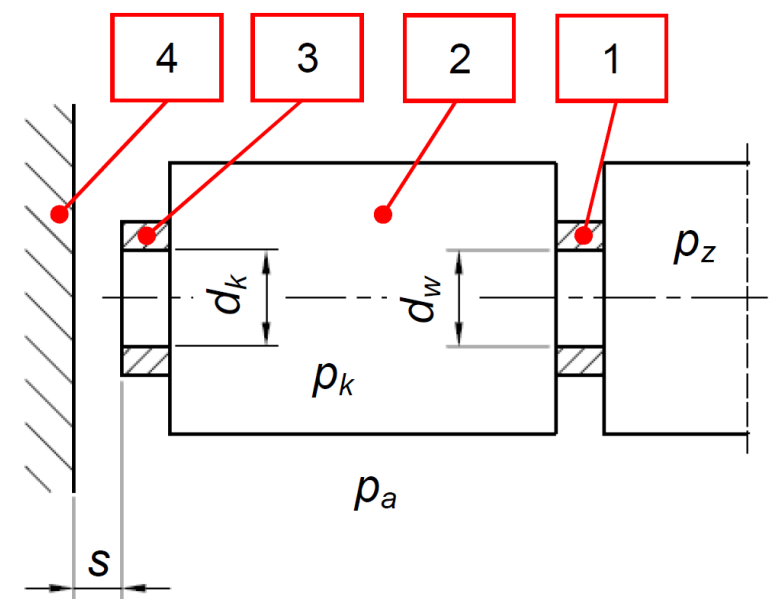

b)

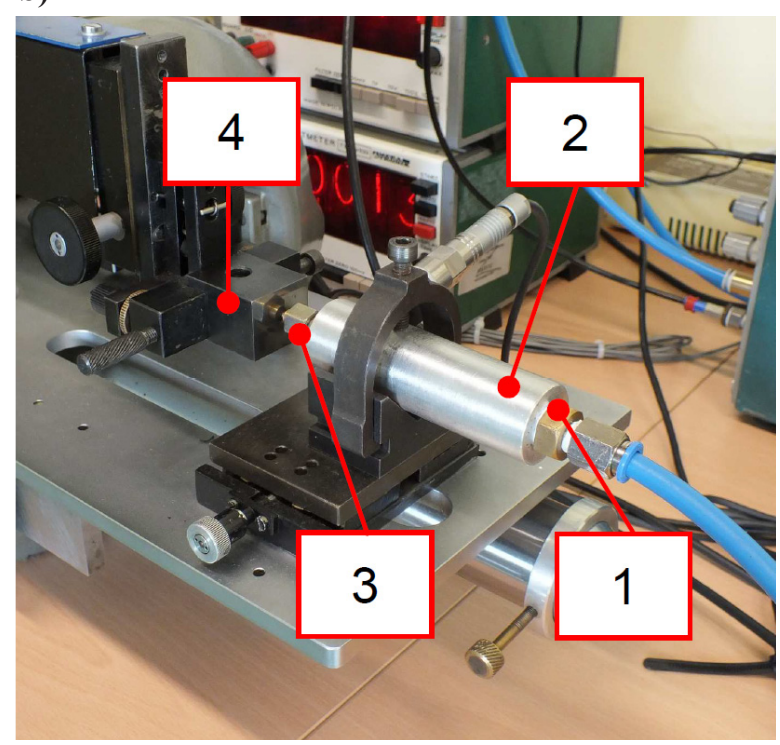

Fig. 1. Typical high-pressure open jet type air gauge: a) scheme; b) view $[1,3,5,8]$ 
a)

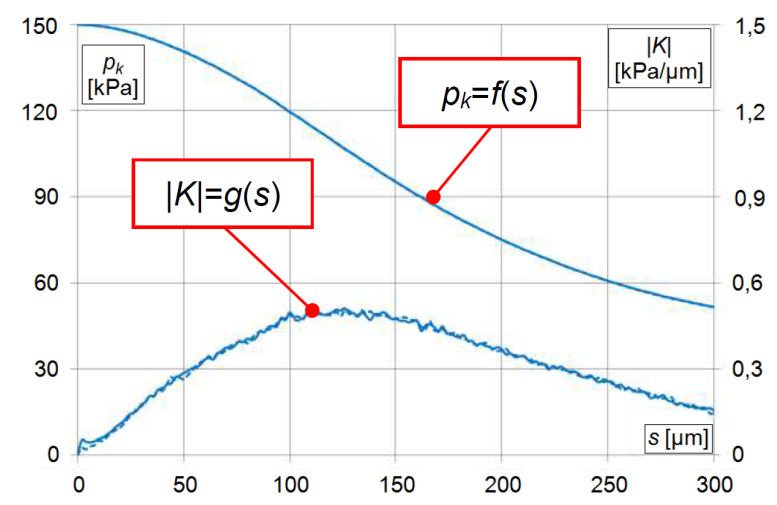

b)

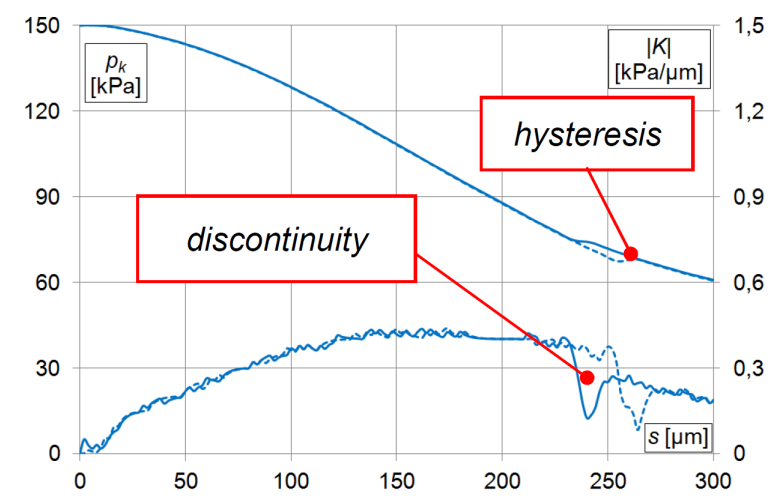

Fig. 2. The static characteristic $p_{k}=f(s)$ and the sensitivity characteristic $|K|=g(s)$ of an air gauge: a) $d_{k}=1.20 \mathrm{~mm}, d_{w}=0.80 \mathrm{~mm}$, b) $d_{k}=1.60 \mathrm{~mm}, d_{w}=0.80 \mathrm{~mm}$

\section{MEASUREMENT SYSTEM TO DETERMINE THE METROLOGICAL PROPERTIES OF AIR GAUGES}

\section{System structure}

When designing the structure of the system, attention was drawn particularly to the possibility of an automatic registration of metrological parameters of air gauges as well as the possibility of a quick change of the type of a tested gauge thanks to an easy exchange of elements such as an inlet and a measuring nozzle as well as a measuring chamber $[1,3,5]$.

The structure of a system includes first-class components. Moreover, the whole system is controlled by a PC.

The measuring system presented in this article is composed of the following three systems:
- the mechanical system;

- the pneumatic system;

- the electronic system.

Figure 3 shows the view of the measuring system for determining the metrological parameters of air gauges.

It consists of: 1 - computer PC; 2 - step motor with epicyclic gearing; 3 - analog manometer; 4 voltmeter V530; 5 - thermal mass flow meter PAT $88 ; 6$ - piezoresistive pressure transducer Kistler 4043 A5; 7 - digital manometer; 8 - amplifier Kistler 4801 A; 9 - pressure stabilizer Aerocon B4; 10 - gauge readout Tesa TT500; 11 - sliding table; 12 - inductive axial movement gauge head Tesa GT21HP; 13 - examined air gauge; 14 - thermocouple; 15 - thermal anemometry probe; 16 - flow meter SMC PFM511/25/50; 17 - control unit; 18 - thermocouple; 19 - restriction valve with the filter SMC AW20-A [6].

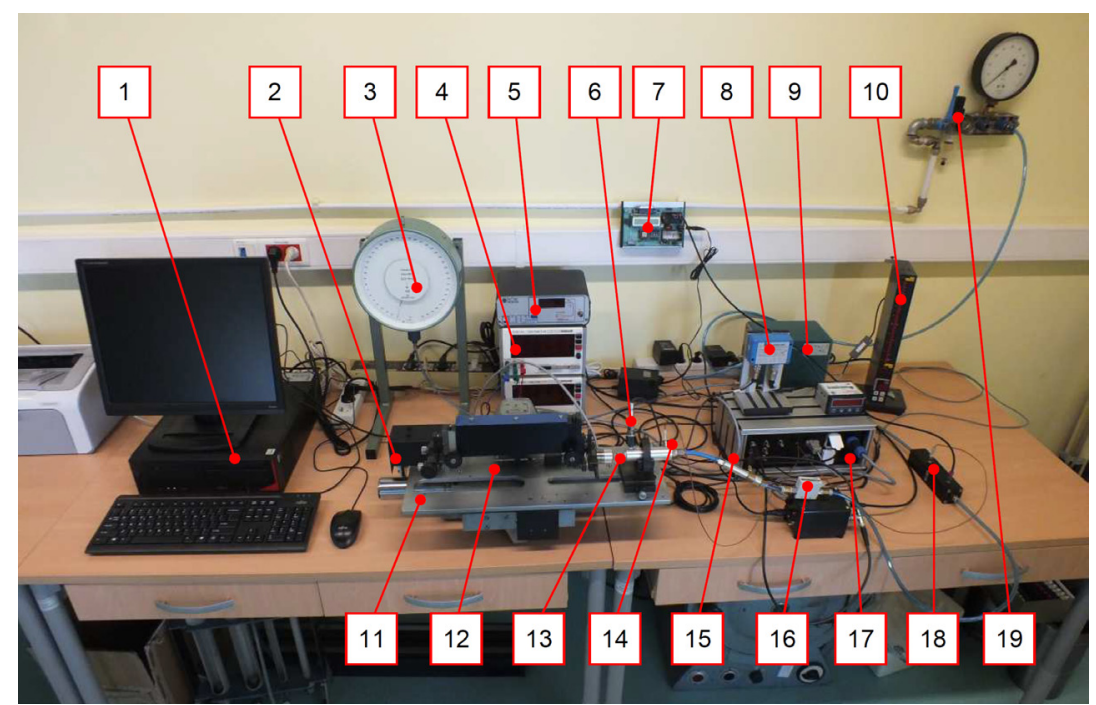

Fig. 3. The view of the measuring system for determining the metrological parameters of air gauges [6] 
The mechanical system is composed of a table with a pneumatic cascade that constitutes an air gauge. The table makes it also possible to change the location of a flapper allowing the change of gap $s$ in the function, in which the cascade pressure $p_{k}$ is measured. The flapper has an angle inclination regulation towards the head of a measuring nozzle, which facilitates the precise adhesion of a nozzle to a flapper.

A gear placed in the table makes it possible to change circular motion to a linear movement. Circular motion is realized through a stepper motor with a planetary gear with 1:36 reduction. It allows the flapper to move in a linear manner smoothly at 1 to $5 \mu \mathrm{m} / \mathrm{s}$ within 0 to $400 \mu \mathrm{m}$. A stepper motor is connected to an electronic system, which makes it possible to control its work with the help of the designated software. The displacement is measured by an inductive axial movement gauge head manufactured by Tesa (length GT21HP, uncertainty $U_{0.95}= \pm 0.17 \mu \mathrm{m}$ ). The gauge is connected to the Tesa TT500 gauge readout, which sends the value of the current placement of a flapper to the control system. The transmission is based on RS-232 standard.

Figure 4 show bloc diagram of the measuring system for determining the metrological parameters of air gauges.

With the help of a thermal anemometry probe installed on a cord that supplies power the transducer, the precision of adhesion of the head of a measuring nozzle to the surface of a flapper for a gap $s-0 \mu \mathrm{m}$ is checked.

Figure 5 shows the bloc diagram of a pneumatic system. The described system is powered by the air coming out of a pneumatic main whose pressure is between 0.6 to $1 \mathrm{MPa}$. The compressed air flows through the system that prepares the air. It is filtered there and separated from oil and its pressure is initially stabilized at $500 \mathrm{kPa}$. The air prepared in this way flows through a precise pressure stabilizer, where its value is set at the level necessary for supplying power to the examined pressure transducer pz. The stabilizer that was used makes it possible to stabilize the pressure within 0-200 kPa range, however, the feed pressure that is most often used for industrial purposes is $\mathrm{pz}-150 \mathrm{kPa}$. Then the air flows through the chamber, where the temperature of the air that powers the transducer is measured. An air flow meter installed in the system measures the flow volume of air qv. The station was equipped with three replaceable air flow meters manufactured by SMC (ranges: $0-25 \mathrm{l} / \mathrm{h}, 0-50 \mathrm{l} / \mathrm{h}$ and $0-100$ $1 / h)$. It enables the researcher to choose the right range depending on the parameters of an examined air gauge. Each of air flow meters generates an analog signal in the $1-5 \mathrm{~V}$ range that is registered by the software.

The compressed air from an air flow meter flows to an air gauge. Gauge structures may differ but the test station makes it possible to apply various structural solutions of air gauges. The most common solution is, however, the structure based on a straight cascade presented earlier (Fig. 2). The station is equipped with a standard measuring chamber, which makes it possible for the inlet and measuring nozzles to be replaced. Because of this, a quick reinforcement of a chamber and the measurements of air gauges of various metrological parameters are possible. The Kistler pressure transducer (range 0-200 kPa) was used to deter-

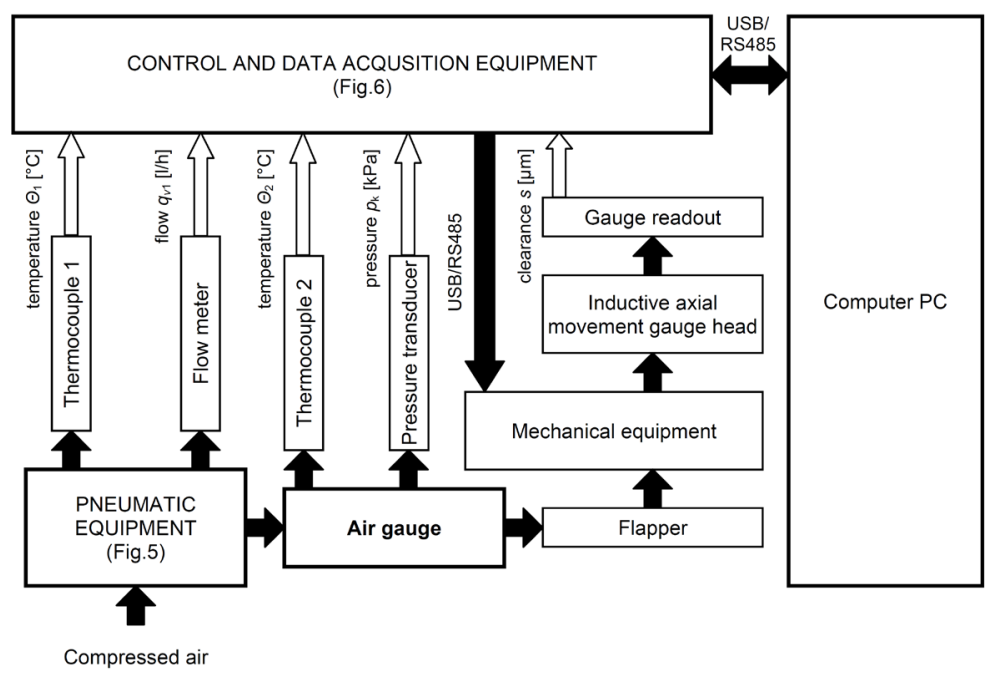

Fig. 4. The block diagram of the measuring system for determining the metrological parameters of air gauges [6] 


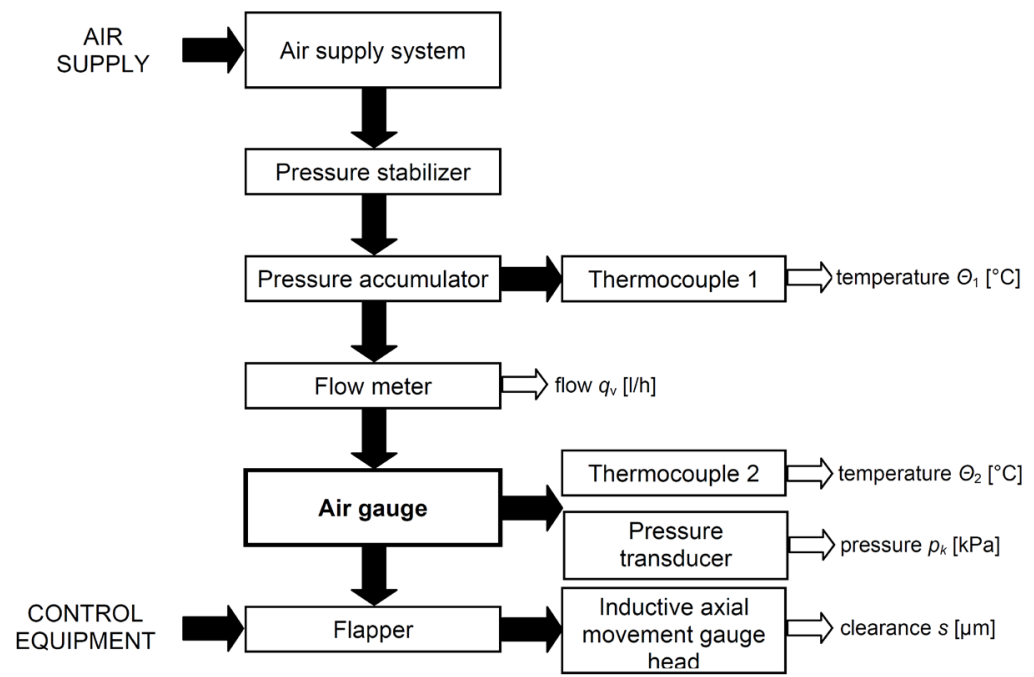

Fig. 5. The block diagram of pneumatic path of the measuring system (pneumatic equipment)

mine the measuring pressure. The temperature of the compressed air is also measured in the chamber. The measurement is performed with the use of a thermocouple. The change of gap s is regulated by the above-mentioned table with a gear and a stepper motor. All the parameters describing the flow of air through the pneumatic system such as: the pressure pk, the flow $q_{v}$, the temperature $\Theta_{1}$ and the temperature $\Theta_{2}$ are registered in the function of the displacement of flapper s.

The electronic system is the next element of the whole system. It was placed in a separate casing made of aluminum sections. On the front panel of the casing there are test signal connec- tors, a gauge readout, a stepper motor connector and a USB connector to connect with the PC. On the back panel there is a mains electricity connector and sockets to supply power to the external components of the system.

Figure 6. shows the structure of the electronic system. The basic component of the electronic system is a card manufactured by Advantech equipped with a 6-channel analog-to-digital converter. The card registers test signals from a pressure transducer, an air flow meter and a strain gauge bridge circuit. This bridge is used for the configuration of a device. The configuration makes it possible to measure the force of a stream

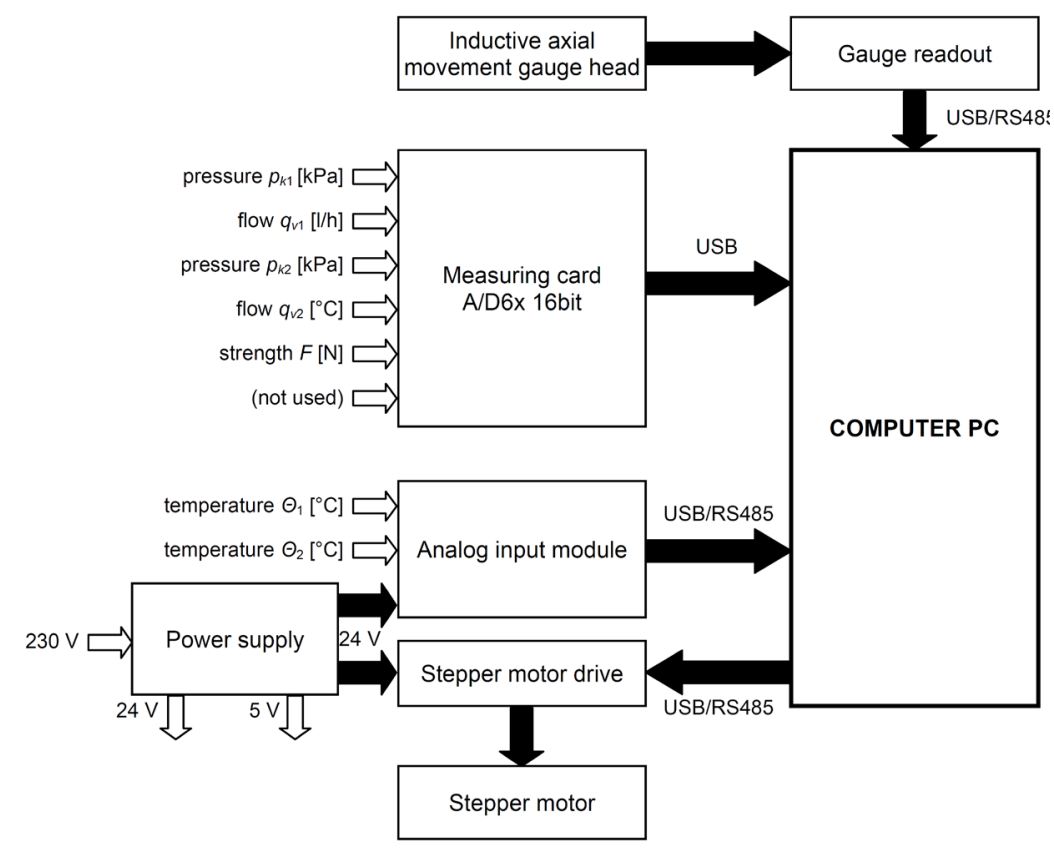

Fig. 6. The block diagram of the control and data acquisition equipment 
of air that comes out. The card is connected to the computer by way of the USB interface. The next component of the electronic system is the Advantech analog input module. It is a device that is designed to register the signals from the thermocouples, including type $\mathrm{T}$ thermocouples, which were used in the system. Two thermocouples measuring the temperature of the air at the intake of the transducer and in the measuring chamber were connected to the module. The data from the module is transmitted by way of a USB/RS485 transducer to the computer.

The Trinamic stepper motor driver is responsible for the work of the stepper motor. It is an integrated driver, which interprets signals transmitted by the RS-485 transducer and uses the data transmission protocol specified by the manufacturer. It has a built-in microprocessor system responsible for generating impulses (steps) and a built-in power controller responsible for controlling two-phase motors of $2 \mathrm{~A}$ per phase. Thanks to the driver one has a full control over the motor (step division, supply current or the holding moment), which makes it possible to precisely regulate the position of the table as well as its speed.

A gauge readout manufactured by Tesa is a separate element of the whole electronic system. The inductive axial movement gauge head, which was described earlier, is connected to the gauge readout. This gauge measures the change of location of the flapper s. Then, this value is transmitted by the RS-485/USB transducer to the computer in the form of the ASCII code.

A standard PC available on the market is responsible for controlling the whole system.

\section{Software}

The Division of Metrology and Measurement Systems of the Poznan University of Technology designed a special software whose responsible for controlling the whole system. Figure 7 shows the window of the software.

This software was written in VB.NET and IDE - the languages developed by Microsoft. The software has intuitive user interface that allows a person to control the measurements and track given values on-line.

After installing the air gauge in question, one has to input its geometrical parameters and the type of the nozzle to the system. Predefined field parameters for circular and slot nozzles were implemented. For non-standard nozzles the comment field has to be used to save data about the geometry of an air gauge in question. To carry out the measurements one has to begin with regulating the position of the flapper so that the speed of the flow measured with a proper thermal anemometry probe would equal $0 \mathrm{~m} / \mathrm{s}$ in the position $s=0 \mu \mathrm{m}$ and the pressure in the measuring chamber would equal the supply pressure. The screws located in the flapper are responsible for the regulation.

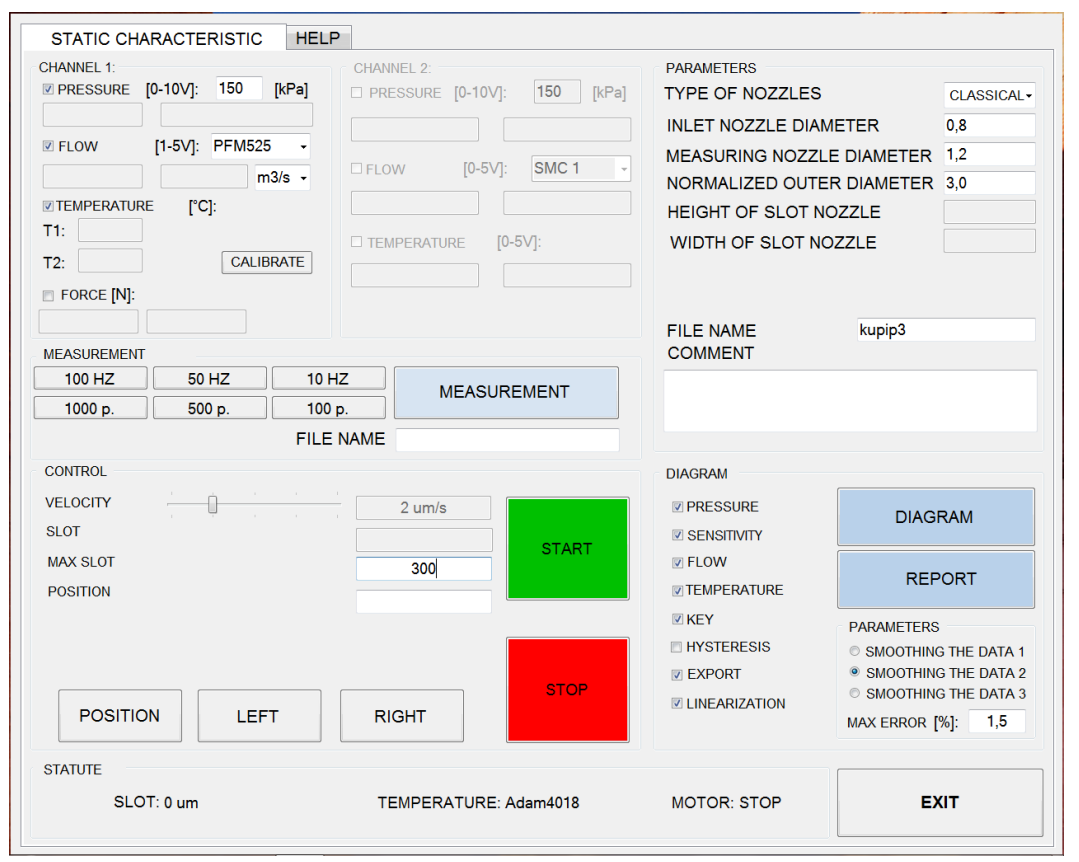

Fig. 7. The window of the software that controls the measurement system 
After inputting the initial parameters for the measurement, the maximum gap for the measurement must be defined. The measurement will be taken for the gaps ranging from the current one to the maximum orifice defined by the user. The measurement data is gathered when the table moves in both directions as the flapper moves away and towards the head of a measuring nozzle. A given number of repetitions of a measurement can also be defined. In this case the measurement will be $n$-fold for the currently installed gauge. There is a possibility of choosing the speed of the table ranging from 1 to $5 \mu \mathrm{m}$. The selection may be done with resolution $1 \mu \mathrm{m}$. After defining all parameters, the measurement is initiated by clicking "START."

Collecting measurement data for particular values of the gap s is initiated by the data transmission from the gauge readout. Raw data is registered every $0.2 \mu \mathrm{m}$ irrespective of the speed of the measurement. For every gap value the proper pressure, flow and temperatures value are assigned. The data is saved in the form of a table in the computer memory, and then as a text file in the location specified by the software on the computer's hard disc. I this way initial data can be later controlled.

\section{RESULTS OF MEASUREMENTS}

After the end of the measurement, the program processes raw data using a defined algorithm (Fig. 8). Registered measurement data is subject to interpolation and then particular measurements are added up. The polynomial smoothing is the next step.

On the basis of the measurement data prepared in such a way, the software makes it possible to calculate the metrological parameters and present the results of the measurements in the form of a graph (Fig. 9). The parameters of an air gauge are calculated on the basis of the coordinates of points subject to linearization for a growing value of the linearity error $\delta_{l}$.

The static characteristic $p_{k}=f(s)$, the flow characteristic $q_{v}=j(s)$, the sensitivity characteristic $|K|=g(s)$, and temperature characteristic $\Theta=h(s)$ can all be presented depending on the need of the user on one graph or each characteristic separately.

The user also gets a table with values of particular points presented on graphs and the values of measuring range $z_{p}[\mu \mathrm{m}]$, sensitivity $K[\mathrm{kPa} /$ $\mu \mathrm{m}]$, initial and end coordinates $s_{p}[\mu \mathrm{m}]$ and $s_{k}[\mu \mathrm{m}]$ and respective measurement pressure $p_{k-} s_{p}$ [kPa] and $p_{k-} s_{k}[\mathrm{kPa}]$ (Fig. 9). This information is vital in the process of designing and assessing air gauges.

Each of the measurements is saved on the disc in the form of a txt file that has a unique iden-

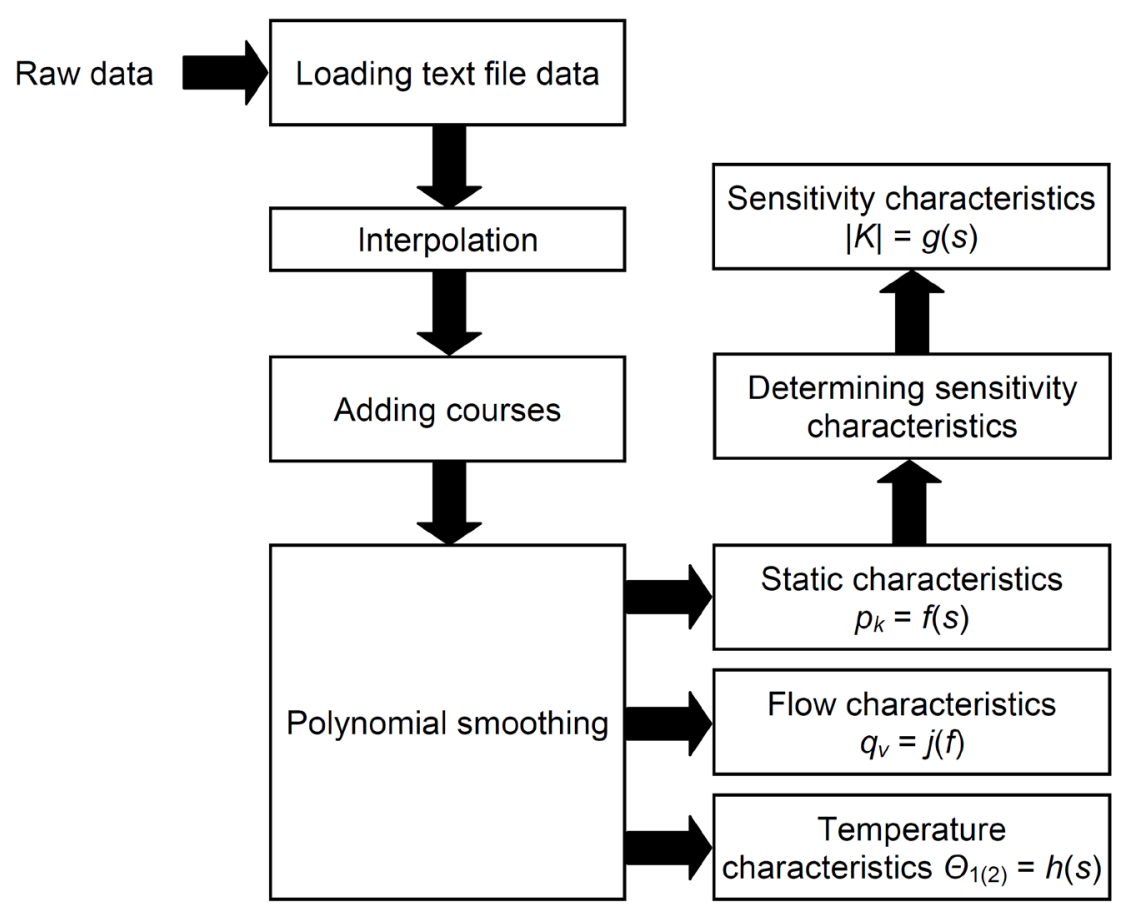

Fig. 8. Data analysis algorithm 


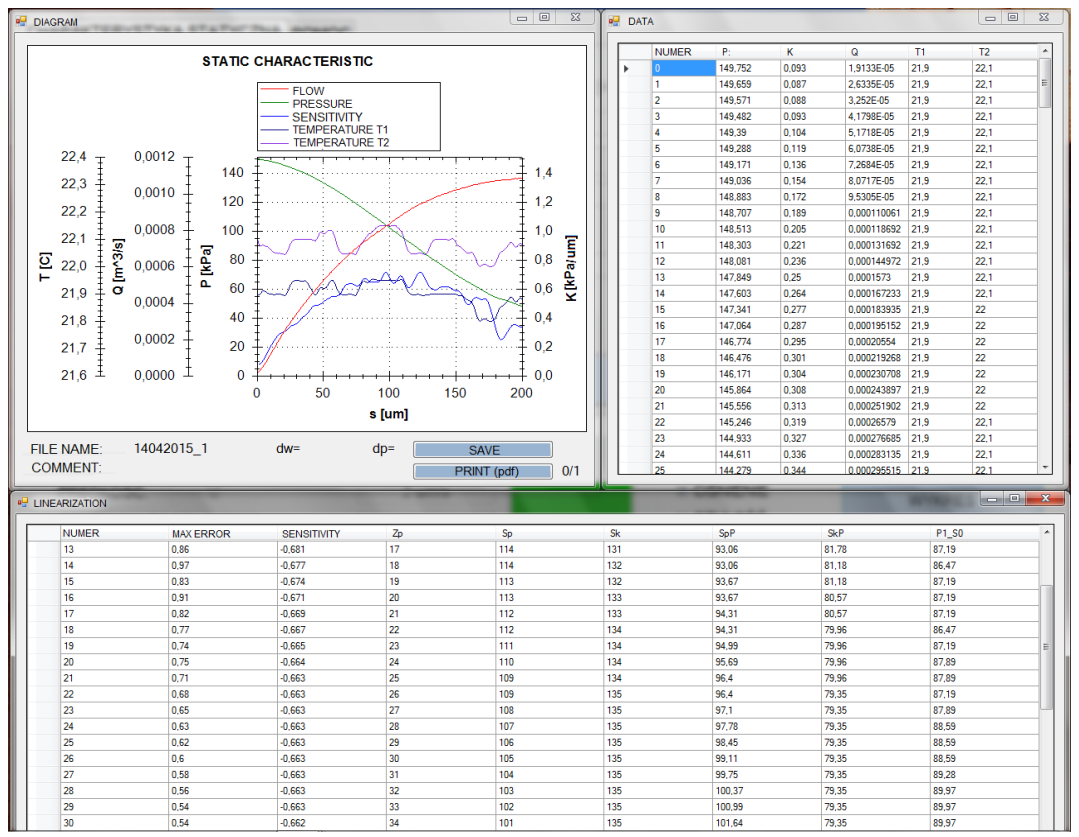

Fig. 9. Sample characteristics and values of parameters

tification number. Thanks to this unique number the user can export the data to other data analysis software. There is also a possibility of generating a report from a single or numerous measurements in the PDF format.

\section{CONCLUSION}

The scientific research presented in this article was prepared in order to determine the metrological parameters of air gauges. The sensors used in the system analyzed in this article allow the scientist to take measurements in a wide scientific spectrum, which guarantees a complex analysis.

Researching new structures of air gauges and determining their metrological parameters with the help of a designed station will make it possible to widen the application of air gauges in static and dynamic control systems.

\section{Acknowledgements}

The presented research results, executed under the subject of No $02 / 22 / \mathrm{DSMK} / 1330$, were funded with grants for education allocated by the Ministry of Science and Higher Education in Poland.

\section{REFERENCES}

1. Dereżyński, J., Jakubowicz, M., Verification tests of the air gauges metrological characteristics, Mechanik, 3(89), 2016, 196-199.

2. Destefani J., Air gauging, Manufacturing Engineering, 4(131), 2003, 5-9.
3. Farago F.T., Curtis M.A., Handbook of Dimensional Measurement, Industrial Press Inc, New York, 2004.

4. Jakubowicz M., Jermak Cz.J., Measurement uncertainty of air gauges for length measurement, Machine Engineering, 3(18), 2013, 48-59.

5. Jermak, Cz.J., Jakubowicz, M., Dereżyński, J., Rucki, M., Air Gauge Characteristics Linearity Improvement, Journal of Control Science and Engineering, 2016, , Article ID 8701238, 7 pages, http://dx.doi.org/10.1155/2016/8701238

6. Jermak, Cz.J., Jakubowicz, M., Dereżyński, J., Rucki, M., Back-pressure uncertainty estimation for the air gauge experimental rig, Proc. of International Conference on Innovative Technologies, IN-TECH 2016, Prague, Czech Republic 2016, 197-200.

7. Jermak, Cz.J., Rucki, M., Air Gauging: Still Some Room for Development, AASCIT Communication, 2(2), 2015, 29-34.

8. Jermak, Cz.J., Rucki, M., Static Characteristics of Air Gauges Applied in the Roundness Assessment, Metrolology and Measurement Systems, 1(23), 2016, 85-96.

9. Liu J., Pan X., Wang G., Chen A., Design and accuracy analysis of pneumatic gauging for form error of spool valve inner hole, Flow Measurement and Instrumentation, 23, 2012, 26-32.

10. Rucki M., Barisic B., Szalay T., Analysis of air gage inaccuracy caused by flow instability, Measurement, 6(41), 2008, 655-661.

11. Schuetz G., Pushing the Limits of Air Gaging-And Keeping Them There, Quality Magazine, 7(54), 2015, 22-26.

12. Tanner, C.J., Air gauging - history and future developments, Institution of Prod. Eng. J., 7(37), $1958,448-462$. 